\title{
Flutter prediction for a very flexible wing wind tunnel test
}

\author{
Norberto Goizueta*, Andrew Wynn ${ }^{\dagger}$ and Rafael Palacios \\ Department of Aeronautics, Imperial College London, SW7 2AZ \\ Ariel Drachinsky ${ }^{\S}$ and Daniella E. Raveh ${ }^{\mathbb{I}}$ \\ Faculty of Aerospace Engineering, Technion - IIT, Israel
}

Paper for Special Session: Progress of the 3rd Aeroelastic Prediction Workshop Large Deflection Group

\begin{abstract}
Two different nonlinear aeroelastic tool sets, SHARPy and the Modal Rotation Method (MRM), have been employed to predict and design a wind tunnel flutter test campaign of a very flexible wing, the Pazy Wing, as part of the 3rd Aeroelastic Prediction Workshop. The first method, SHARPy, uses geometrically exact beams coupled with an Unsteady Vortex Lattice, which is linearised about a deformed configuration, reduced by means of Krylov subspaces and analysed to compute the stability boundaries of the wing. The MRM is based on structural modal data, from either beam models or finite element models, coupled with a doublet-lattice aerodynamic model from ZAERO of the straight wing configuration. The excellent agreement between numerical and experimental data for structural-only and static aeroelastic analyses paves the way for predicting the stability boundaries of the Pre-Pazy wing with sufficient confidence for the safe design of a flutter wind tunnel test campaign.
\end{abstract}

\section{Introduction}

S OLAR-powered, high-altitude, long endurance aircraft (HALE) have become a reality in the aerospace industry with Sthe development of aircraft such as Airbus' Zephyr [1], Boeing's Odysseus [2], BAE Systems' PHASA 35 [3] or Skydweller [4]. All these concepts seek perpetual endurance, stratospheric flight for communication or surveillance - in essence, pseudo-satellite - missions. Perpetual, year-round endurance particularly imposes constraining requirements on the design, demanding extreme aerodynamic efficiency and very lightweight structures. The former leads to designs with very high aspect ratio, that coupled with modern lightweight materials result in wings that are capable of achieving deformations comparable to their span.

The large deformations induced by the aerodynamic forces make it indispensable to design the system from an aeroelastic perspective, with tools and methods capable of capturing nonlinear effects caused by such large deformations. This was a clear outcome of the Helios mishap in 2003 [5], yet is still an area where active research is required, as shown by the Google Solara crash in 2015 [6].

Although full three-dimensional finite element models and computational fluid dynamics solvers that apply full Navier-Stokes equations can be coupled together to offer a complete aeroelastic solution, the computational cost is prohibitive. Thus, the need exists to turn to medium-fidelity models that can offer sufficient accuracy at a fraction of the cost, in particular if control-related applications, which are highly sensitive to model size, are desired down the line. Therefore, provided that structural members are often very slender in these aircraft, one-dimensional beam approximations are an attractive alternative for the structural model. In addition, the laminar, mostly-attached nature of the flow and the prevalence of thin lifting surfaces make potential flow based aerodynamics a viable option [7].

However, as with all computational methods, and in particular for these medium-fidelity methods, the question remains as to how accurate are they and whether they are able to capture complex phenomena. Few aeroelastic benchmark cases for which experimental data is available exist and thus most comparisons between methods have been purely numerical [8]. In terms of experimental flutter data sets, the Dowell wing [9] in 2001 is a notable example. However, the data available is reduced and limited to a few angles of attack. Recently, a novel benchmark case has been presented by the research group at Technion [10] of a very flexible clamped wing for which a flutter test campaign has been planned.

\footnotetext{
*PhD Student, CAGB 308, South Kensington Campus. (norberto.goizueta13@imperial.ac.uk)

† Senior Lecturer in Control, CAGB 340, South Kensington Campus. (a.wynn@imperial.ac.uk)

$¥$ Professor of Computational Aeroelasticity, CAGB 338, South Kensington Campus. AIAA Associate Fellow (r.palacios@imperial.ac.uk)

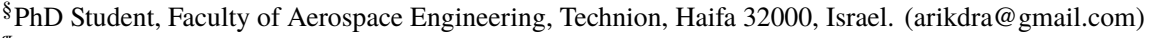

IIProfessor, Faculty of Aerospace Engineering, Technion, Haifa 32000, Israel. AIAA Associate Fellow (daniella@technion.ac.il)
} 
This wing, named the Pazy wing, consists of an aluminum spar with Nylon, evenly-spaced, NACA0018-shaped ribs and a wing tip rod. To give it the external aerodynamic shape, the wing is covered with an Oralight skin.

This paper aims to compare and assess the performance of two medium-fidelity aeroelastic models - SHARPy [11] and a Modal Rotation Method (MRM) [12] — against the currently available experimental results and provide predictions for flutter that may be used in the design of further wind tunnel experiments. We present in Sec. II both methodologies, which are applied to the Pazy wing that is thoroughly described in Sec. III alongside the relevant approximation models. Section IV compares the numerical models against the static experimental results. The strong correlation between both sets paves the way for a on-going flutter prediction exercise, discussed in Sec. V.

This activity is part of the Large Deflection working group of the 3rd Aeroelastic Prediction Workshop (AePW3) led by NASA. This project aims to bring together different numerical and experimental knowledge from academia to solve relevant and modern aeroelastic problems that may be used as benchmark for validation of future tool sets. For such purpose, we provide all models, scripts and codes used to obtain the results presented herein available as open source. Details on how to obtain them can be found in the Research Data Appendix.

\section{Numerical Methods}

Two computational methods have been used with different structural and aerodynamic models. Both methods can be considered to be of medium fidelity and are tailored for aeroelastic applications such as the one at hand. Thus, this section will provide a brief summary and direct the reader to the appropriate references for further detail.

\section{A. SHARPy}

SHARPy (Simulation of High Aspect Ratio aeroplanes and wind turbines in Python) is a nonlinear aeroelastic simulation toolbox available under open-source licence [11]. At its core, SHARPy couples a nonlinear beam solver with an unsteady aerodynamic model based on potential flow assumptions. Additional modules focus on the linearisation of these nonlinear models around nonlinear static equilibria and model reduction. SHARPy has tens of modules that can be combined to offer various analysis capabilities and interested readers can find details in the extensive online documentation F. For brevity, we will only summarize here those modules that need to be employed to predict flutter of highly-flexible, clamped structures. For this we will (i) solve for the static aeroelastic equilibrium condition of the wing; (ii) linearize the structural and aerodynamic systems about this reference; (iii) reduce the system using modal reduction and Krylov-based methods, respectively; and (iv) analyze the stability of the resulting reduced linear state-space.

The structural model is based on a geometrically-exact 1D beam formulation, with linear constitutive relations and nonlinear velocity and displacement kinematic relations. The formulation is parametrised in displacements and rotations and applied by discretizing the beam in quadratic (3-node) finite elements [13, 14]. The nonlinear equations that result from the application of Hamilton's principle take the form of [8]

$$
\mathcal{M}(\boldsymbol{\eta}) \ddot{\boldsymbol{\eta}}+\boldsymbol{Q}_{\mathrm{gyr}}(\boldsymbol{\eta}, \dot{\boldsymbol{\eta}})+\boldsymbol{Q}_{\mathrm{stiff}}(\boldsymbol{\eta})=\boldsymbol{Q}_{\mathrm{ext}}(\boldsymbol{\eta}, \dot{\boldsymbol{\eta}}),
$$

where $\mathcal{M}$ is the mass matrix and $\boldsymbol{Q}$ represents the discrete external (aerodynamic), gyroscopic and stiffness forces. The flexible degrees of freedom expressed in a body-attached frame, $\boldsymbol{\eta} \in \mathbb{R}^{6 \times\left(n_{\text {nodes }}-1\right)}$, include displacements and rotations, the latter parametrised through a Cartesian rotation vector (CRV).

These equations can be linearised about a reference equilibrium condition $\left(\boldsymbol{\eta}_{0}, \dot{\boldsymbol{\eta}}_{0}\right)$ where $\ddot{\boldsymbol{\eta}}_{0}=\mathbf{0}$, under the assumption of small perturbations to the flexible degrees of freedom [15]. The resulting equations

$$
\mathcal{M}\left(\boldsymbol{\eta}_{0}\right) \delta \ddot{\boldsymbol{\eta}}+\boldsymbol{C}\left(\boldsymbol{\eta}_{0}, \dot{\boldsymbol{\eta}}_{0}\right) \delta \dot{\boldsymbol{\eta}}+\mathcal{K}\left(\boldsymbol{\eta}_{0}, \dot{\boldsymbol{\eta}}_{0}\right) \delta \boldsymbol{\eta}=\delta \boldsymbol{Q}_{\text {ext }}(\delta \boldsymbol{\eta}, \delta \dot{\boldsymbol{\eta}}),
$$

adopt the familiar form, where $\mathcal{C}$ and $\mathcal{K}$ are the constant, tangent damping and stiffness matrices, respectively. The system can then be projected onto modal coordinates (which will be dependent of the equilibrium point) and truncated to retain the modes that capture the most significant dynamics.

In SHARPy, the aerodynamics are solved using an Unsteady Vortex Lattice Method (UVLM) which is based on the assumption of potential flow [7, 8, 16, 17]. Vortex panels are laid out over lifting surfaces, with their spanwise location coincident with that of the underlying structural elements. In turn, the wake can be modelled by either infinitely long horseshoe vortices (in static simulations) or with discrete panels that are influenced by the bound and other wake vortices (for unsteady simulations). The flow field itself is solved enforcing the non-penetration boundary condition

\footnotetext{
*http://imperial.ac.uk/aeroelastics/sharpy
} 
at the centre of the bound vortex rings by adjusting their circulation strength.The formulation is nonlinear given the dependency of the aerodynamic influence coefficients (which contain the information on how each vortex affects others) on the instantaneous deformation of the structure and shape of the wake sheet. Once the circulation of the vortices has been solved for, the aerodynamic forces are calculated attending to steady and unsteady contributions: the former is calculated using the Joukowsky theorem [18] whereas the latter uses Bernoulli's unsteady equation based on the time derivative of the circulation, which is calculated by finite differences. An important underlying assumption is that all viscous effects are confined to thin boundary layers whose effects can be neglected, including viscous drag contributions and flow separation [7]. Stall has not been modeled.

The linearization of the UVLM equations is performed analytically about the deformed shape under the assumption of constant aerodynamic influence coefficients (i.e. small structural deformations) and a frozen, yet arbitrary, wake shape [19]; the linearization includes steady load effects [20]. To achieve convergence and capture unsteady effects a highly discretized bound vortex lattice and a long wake are required [19, 21, 22], thus it is highly convenient to reduce the system to alleviate the computational burden of linear analysis tools (i.e computing eigenvalues, etc.). Thus, we turn to Krylov subspace methods to reduce the dimension of the aerodynamic system [23]. This is a computationally efficient model order reduction method based on matching the transfer functions and their derivatives at user-defined frequencies, which by the nature of the UVLM method we limit to reduced frequencies below approximately 0.6 [7]. Nonetheless, since this reduction method is based on transfer function matching, the reduced order system is prone to be of significant size when the input/output dimensionality is large and this is the case of the linear UVLM, where the inputs (displacement and velocities) and outputs (forces) are defined at each of the lattice vertices. Therefore, under the assumption of chordwise rigid rotations of the beam elements, the UVLM input/output space is projected onto the modal coordinates of the underlying beam element, which reduces the number of inputs and outputs to the number of chosen structural modes. Then, the Krylov subspace reduction can be performed efficiently, leading to substantial model size reductions: full order, converged UVLM systems are typically in the range of $O\left(10^{5}\right)$ states and they are reduced to a dimension of $O(10)$ states.

The coupling between the linear structural and aerodynamic models is trivial, since the inputs and outputs of both systems are expressed in the structural modal coordinates. The aeroelastic system is also of a sufficiently small size that stability and other linear analyses can be performed very efficiently.

\section{B. Modal Rotation Method}

A static mathematical model of the MRM was presented in [12], and was recently extended for a dynamic aeroelastic solution in [24]. It is developed for computing nonlinear large deformations of flexible, slender structures. While the MRM is not limited to analyzing beams, it solves the structural deformations along a reference line. For wing structures, this line runs over the wing-span, typically along the mid-chord, the leading or trailing-edge. The modes are described in segments over the reference line. The segmentation and reference line concepts are illustrated in Fig. 1 . The MRM formulation is based on curvature mode shapes that are computed in a free-vibration analysis from a linear finite-element model. The curvature modes are interpolated to segments along the reference line. Provided that this line is divided into sufficiently small segments, the change in the rotation in each segment is small. This allows for a linearized, modal based evaluation of the rotation change in each segment:

$$
\{d \theta\}=\left[\phi_{d \theta}\right]\{\xi\}
$$

and their summation over the structure (over the reference line) using nonlinear kinematics:

$$
\left\{U_{i}\right\}=\left\{U_{i-1}\right\}+\left[R_{i}\right]\left\{d l_{i}\right\}
$$

where $\left[R_{i}\right]$ is a rotation matrix of the $i$-th segment which is based on the summation of the local curvatures (represented via Euler angles) from the root to the segment. To accurately model the internal bending moments, the MRM applies an iterative procedure that updates the location and orientation of the applied external loads based on the deformed shape via external correction moments:

$$
\left\{\Delta M_{i}\right\}=\left\{M_{\mathrm{loc}_{i}}\right\}-\left\{M_{0 i}\right\}-\sum_{j=i+1}^{n}\left\{\Delta M_{j}\right\}
$$

where $\left\{M_{0 i}\right\}$ is the internal moment acting on the undeformed configuration. The MRM is coupled with an aerodynamic model, based on the rigid configuration, to compute static aeroelastic deformations and the flutter velocity about the static equilibrium. Two methods are proposed to update the structural data of the deformed structure and to alter the 


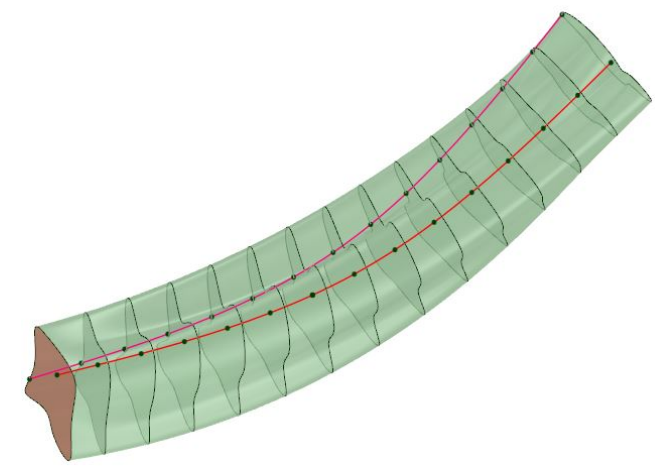

Fig. 1 Illustration of the segmentation and two arbitrary reference lines used in the MRM [12].

unsteady aerodynamic matrices, and to obtain a flutter solution of the deformed wing. One is based on discrete mass and aerodynamic properties of the wing and the other only on modal data, thus providing a nonlinear solution that is based only on linear modal data. The curvature based representation yields a constant generalized stiffness matrix and the updating procedure of the mass and aerodynamic matrices is done by representing the deformed mode shapes as a combination of the basic mode shapes

$$
\left\{\phi_{\text {deformed }}\right\}_{i} \approx \sum_{j=1}^{m} G_{i j}\{\phi\}_{j}
$$

or

$$
\left[\phi_{\text {deformed }}\right] \approx[\phi][G],
$$

thus yielding an expression for the deformed mass matrix that is based directly on the modal mass matrix of the undeformed structure:

$$
\left[G M_{\text {deformed }}\right]=\underbrace{\left[G^{\top}\right]\left[\phi^{\top}\right]}_{\left[\phi_{\text {deformed }}^{\top}\right]}[M] \underbrace{[\phi][G]}_{\left[\phi_{\text {deformed }}\right]}=\left[G^{\top}\right][G M][G]
$$

For the update of the deformed aerodynamic matrices, we assume that, locally, the relation between an incremental deformation (namely, the local incremental angle of attack) and the local forces do not change with the large deformation. This is equivalent to the strip theory assumption that the local strip characteristics are unaffected by the deformation. It is noted that the baseline model does not have to be generated by a strip theory. Under this assumption, updating the aerodynamic matrix is done similarly to the update of the mass matrix, yielding:

$$
\left[Q_{H H, \text { deformed }}(i k)\right]=\underbrace{\left[G^{\top}\right]\left[\phi^{\top}\right]}_{\left[\phi_{\text {deformed }}^{\top}\right]}[A I C(i k)] \underbrace{[\phi][G]}_{\left[\phi_{\text {deformed }}\right]}=\left[G^{\top}\right]\left[Q_{H H}(i k)\right][G]
$$

where $A I C(i k)$ is the discrete aerodynamic influence coefficient matrix, assumed to be unknown explicitly, and $Q_{H H}(i k)$ is the generalized aerodynamic coefficient matrix. Given the deformed, linearized stiffness, mass, and aerodynamic matrices, flutter can be evaluated with any conventional flutter solution technique, such as the $k$-method used in the current case.

\section{Case Study: Pazy Wing}

The Pazy wing [25] is a highly flexible wing, designed recently for wind tunnel aeroelastic experiments to serve as a benchmark for flexible wing studies. The wing model is shown in Fig. 2 It has a chord length of $100 \mathrm{~mm}$, a span of $550 \mathrm{~mm}$, and a NACA 0018 airfoil rib profile. The wing structure is made of an Aluminum 7075 spar, of dimensions $550 \mathrm{~mm}$ (length) by $60 \mathrm{~mm}$ (width) by $2.25 \mathrm{~mm}$ (thickness), and a Nylon PA2200, 3D-printed, chassis. The wing is 
covered with Polyester foil which is typically used in radio-controlled drones. A $300 \mathrm{~mm}$ long and $10 \mathrm{~mm}$ diameter wing-tip rod is 3D printed as part of the chassis. The rod is used for attaching weights (via drilled holes) that can modify the dynamic properties of the structure and alter the flutter speed. Overall, the wing weighs $0.32 \mathrm{~kg}$ without the base with which it attaches to the wind tunnel floor.

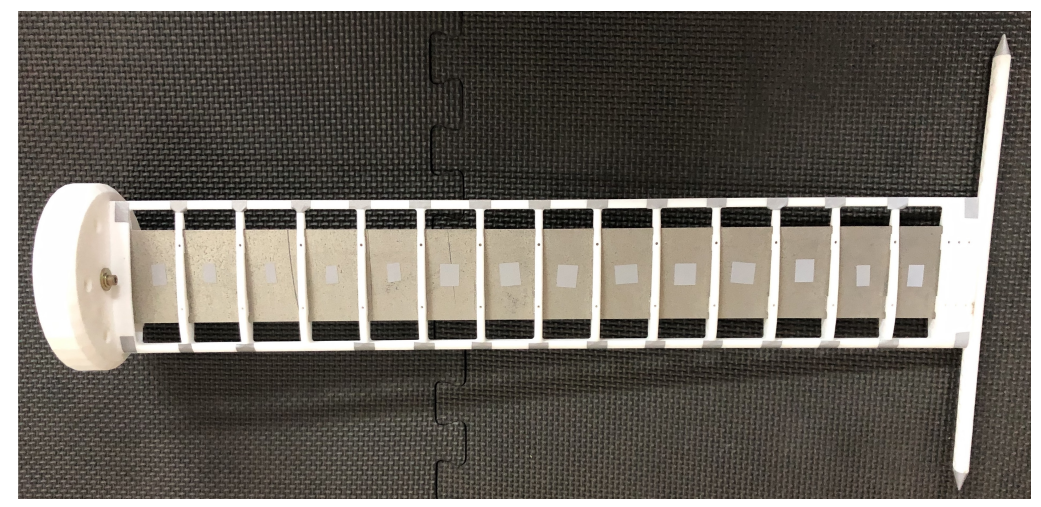

Fig. 2 Pazy wing photo

\section{A. Finite Element Model}

The Pazy wing model analysed computationally in this paper is referred to as Pre-Pazy Wing Model and has been modelled in SolidWorks. The MSC Nastran FE model is shown in Figure 3 . Two different models were constructed: one without the cover and the other, with the cover and additional mass representing the mass of the accelerometer at the tip and the cables. The models have been calibrated against ground vibration test measurements as described in [25].

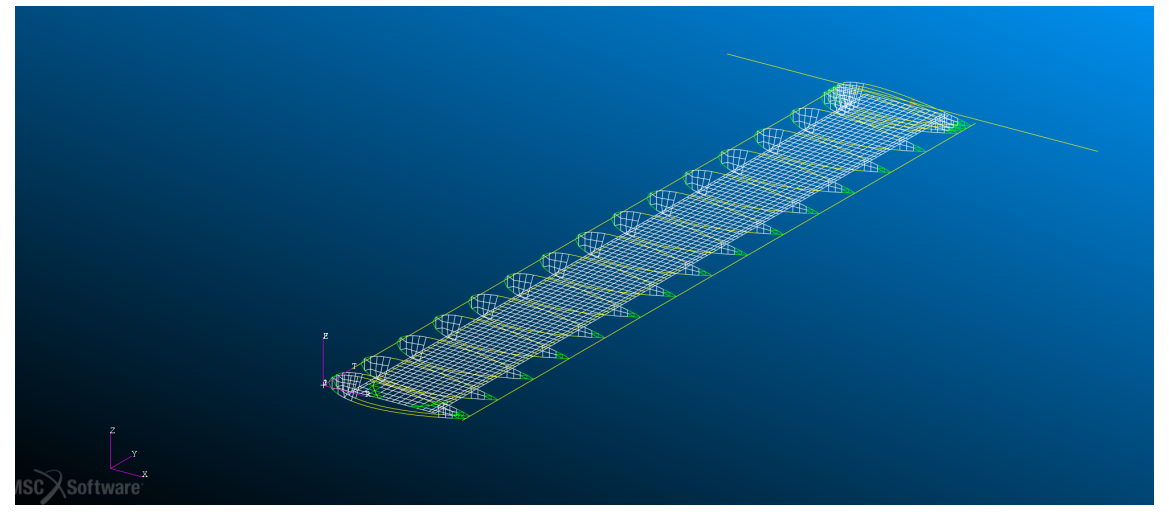

Fig. 3 Finite-element model

\section{B. Model Reduction}

SHARPy's structural module requires of one-dimensional beams represented by 6-by-6 stiffness and mass matrices. To obtain these, the full 3D FEM can be loaded in several directions to obtain the relevant stiffness coefficients that are applicable to one-dimensional beams. This has been done in [26], where Riso et al. developed the beam model for its use in UM/NAST (University of Michigan Nonlinear Aeroelastic Simulation Toolbox). The UM/NAST baseline model has been adapted for SHARPy since in SHARPy the stiffness matrix is a 6-by-6 matrix as opposed to UM/NAST's 4-by-4. The difference being in the shear terms which have been modelled in the SHARPy model as with a large number to simulate zero compliance, although the vibration characteristics are not very sensitive to this term. Both the UM/NAST and SHARPy models share the same beam reference line, located at $44.1 \%$ chord. 
The Pazy wing's Nylon ribs have a NACA0018 airfoil shape which is symmetric, therefore modeled as a flat plate of vortex panels in SHARPy. For static simulations, the wake is modeled by infinitely long horseshoe vortices whereas, for dynamic ones, the circulation shed by the bound vortices is convected downstream from the deformed wing and retained, resulting in a wake sheet of discrete vortex panels of constant circulation that keep a history of the aerodynamic unsteady effects. The aerodynamics of the skin-on and skin-off models are identical.

For consistency between the baseline models, the MRM structural model was based on a coupled 6-by-6 beam model built in Matlab with the stiffness coefficients from [26]. Twenty mode shapes were extracted from 100 FE nodes along the beam and interpolated to 400 segments along the reference line over the wingspan. A similar analysis based directly on the FE model, without the beam reduction can be found in [24]

The MRM aerodynamic model is based on the doublet-lattice method. A rigid aeroelastic analysis was conducted in Zaero [27]. For the static aeroelastic analysis the integrated forces per strip were used to generate a strip model, with the aerodynamic normal force coefficient slope and center of pressure location varying along the span as shown in Fig. 4 For the flutter analysis, the generalized aerodynamic coefficient matrices, $\left[Q_{h h}\right]$, at several reduced frequencies of the undeformed structure were exported from Zaero and used as a database for the flutter analysis of the deformed wing .

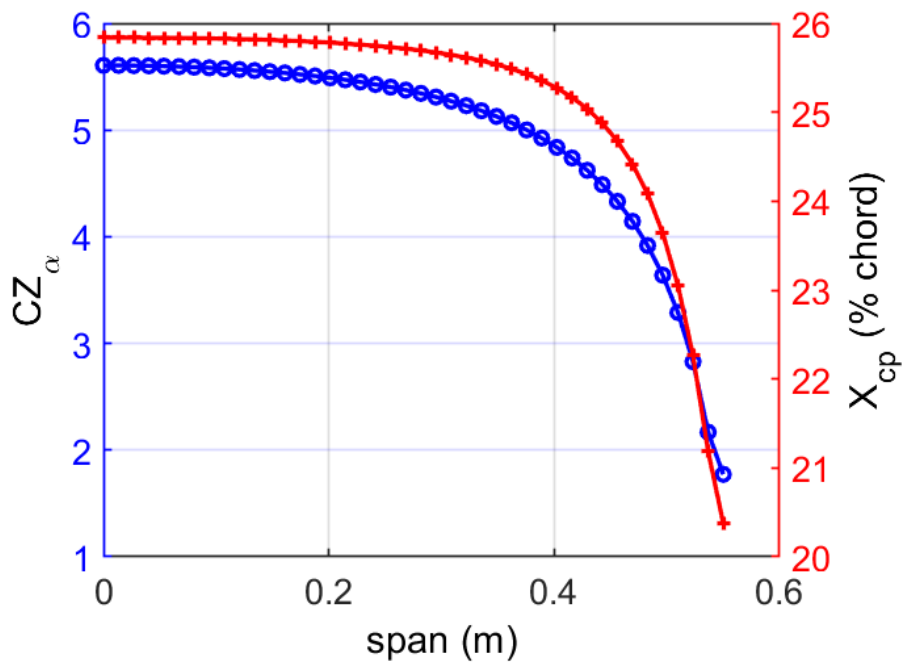

Fig. 4 Span-wise distribution of the sections' normal force coefficient slope and center of pressure locations, computed from Zaero for the undeformed structure.

\section{Modal Analysis}

A modal analysis of the unloaded Pazy wing has been performed to validate the sectional coefficients of the equivalent beam models against the full 3D FEM. Tables 1 and 2 show the original FEM, UM/NAST [26], SHARPy and MRM frequencies. Since the SHARPy and MRM models are derived from the UM/NAST model, the relative error between them and UM/NAST is shown. With a maximum error of $0.7 \%$ between SHARPy and UM/NAST models in the 3rd out-of-plane (OOP) bending mode, the SHARPy model can be considered acceptable, as can the MRM with a maximum $1.96 \%$ difference in the 1st torsion mode. Finally, excellent agreement is observed between the full 3D FEM and the beam models' frequencies.

\section{Static Results}

The static tests performed include structural-only simulations and steady aeroelastic simulations and are used to compare SHARPy and the MRM models. The experimental results shown for both the structural tests and the wind tunnel tests have been obtained from [28]. In addition, throughout the rest of this paper several mentions are made to UM/NAST results [29]; these are used for reference since the beam models employed herein are developed from the UM/NAST one. 
Table 1 Modal frequencies comparison with the skin fitted.

\begin{tabular}{llcccc}
\hline Mode & & NASTRAN [Hz] & UM/NAST [Hz] & SHARPy [Hz] & MRM [Hz] \\
\hline 1st OOP bending & OOP1 & 4.67 & 4.68 & 4.69 & 4.67 \\
2nd OOP bending & OOP2 & 30.68 & 30.82 & 30.87 & 30.69 \\
1st torsion & T1 & 43.57 & 43.51 & 43.71 & 42.66 \\
3rd OOP bending & OOP3 & 87.97 & 88.82 & 88.19 & 87.64 \\
1st IP bending & IP1 & 112.88 & 113.92 & 113.48 & \\
\hline
\end{tabular}

Table 2 Modal frequencies comparison without the skin.

\begin{tabular}{llcccc}
\hline Mode & & NASTRAN [Hz] & UM/NAST [Hz] & SHARPy [Hz] & MRM [Hz] \\
\hline 1st OOP bending & OOP1 & 4.42 & 4.42 & 4.43 & 4.41 \\
2nd OOP bending & OOP2 & 29.02 & 29.11 & 29.15 & 28.99 \\
1st torsion & T1 & 41.53 & 41.44 & 41.63 & 40.63 \\
3rd OOP bending & OOP3 & 83.34 & 83.92 & 83.34 & 82.78 \\
1st IP bending & IP1 & 112.56 & 113.53 & 113.11 & \\
\hline
\end{tabular}

\section{A. Bending structural tests}

Static bending tests have been performed to compare the stiffness properties of the equivalent beam models to the UM/NAST model from which the properties have been extracted, its respective parent FEM analysed in MSC NASTRAN and against experimental results [10].

The bending test is performed by placing a dead load at the wing tip at the mid-chord position. Numerical results obtained from the nonlinear modules in SHARPy and using MRM are shown in Fig. 5 which are compared against the experimental data set and the UM/NAST and NASTRAN results [29]. The offset between the experimental and numerical data is due to initial jig shape deformation as the Pazy wing is subject to manufacturing tolerances. This geometrical initial deformation causes a slight decrease in bending stiffness compared to the straight wing models, as shown by the increased compliance gradients in Table 3 between experiment/MRM and the SHARPy results.

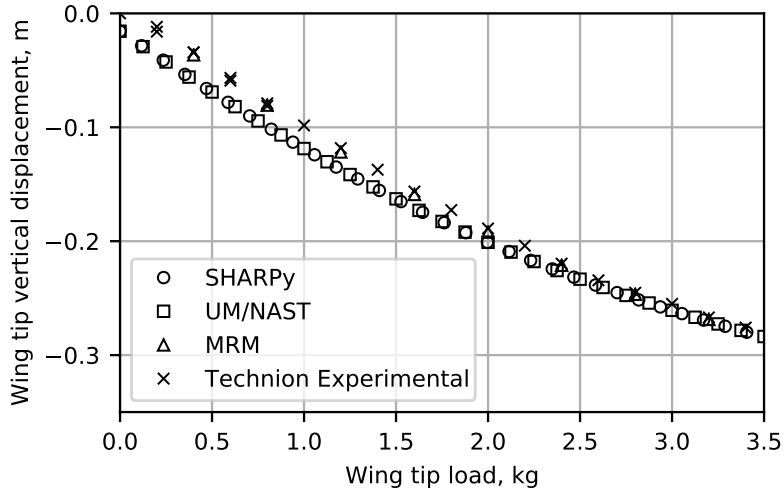

(a) Skin on

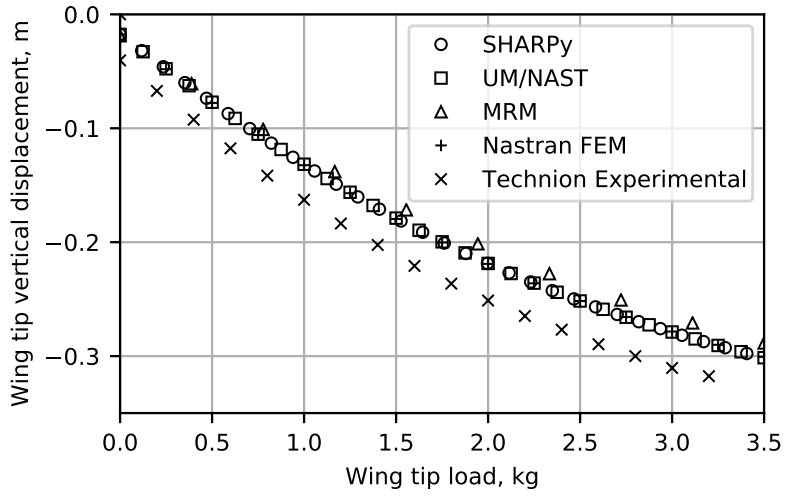

(b) Skin off

Fig. 5 Wing tip, mid-chord vertical displacement comparison under a dead load between SHARPy, MRM, UM/NAST and Technion's experimental results. 
Table 3 Linear bending curve slope and intercept for a linear regression coefficient of 0.999 for the bending test up to $1 \mathrm{~kg}$ load.

\begin{tabular}{lcccc}
\hline & \multicolumn{2}{c}{ With skin } & \multicolumn{2}{c}{ Without skin } \\
& Gradient $[\mathrm{cm} / \mathrm{kg}]$ & Intercept $[\mathrm{cm}]$ & Gradient $[\mathrm{cm} / \mathrm{kg}]$ & Intercept $[\mathrm{cm}]$ \\
\hline SHARPy & -10.35 & -1.67 & -11.46 & -1.90 \\
UM/NAST & -10.35 & -1.67 & -11.46 & -1.90 \\
MRM & -11.23 & 0.90 & -10.57 & 1.91 \\
Experiment & -10.98 & 0.82 & -12.64 & -4.13 \\
NASTRAN & & N/A & -11.65 & -1.81 \\
\hline
\end{tabular}

\section{B. Torsional structural tests}

Torsional tests have been performed on the manufactured wing [10] and compared against the numerical results. This test involved a dead weight being placed at the wing tip, $80 \mathrm{~mm}$ ahead of the leading edge. Note that the wing tip rod, shown in Fig. 2, onto which the load is attached is modeled as a rigid member. Figure 6 shows the wing tip, mid-chord vertical displacement for all models, which are in good agreement, as well as the offset caused by the initial shape as discussed in Sec. IV.A The wing tip twist angle, computed as

$$
\theta=\arctan \left(\frac{z^{\mathrm{LE}}-z^{\mathrm{TE}}}{x^{\mathrm{LE}}-x^{\mathrm{TE}}}\right),
$$

where $z$ and $x$ are measured in an inertial frame and LE and TE refer to the leading and trailing edges, is shown in Fig.77. In the skin-on case, the initial jig shape deformation causes a geometrical stiffening in torsion - as opposed to what occurs in bending - which becomes more evident beyond $1.5 \mathrm{~kg}$ load. In the skin-off case, despite the $2^{\circ}$ offset between the MRM and SHARPy/UM results, the gradient of linear part of the curve (below loads of $1.5 \mathrm{~kg}$ ) shows a good match. Beyond that, the MRM shows a stiffer response. Finally, the agreement between the beam models and the NASTRAN FEM in twist is also excellent.

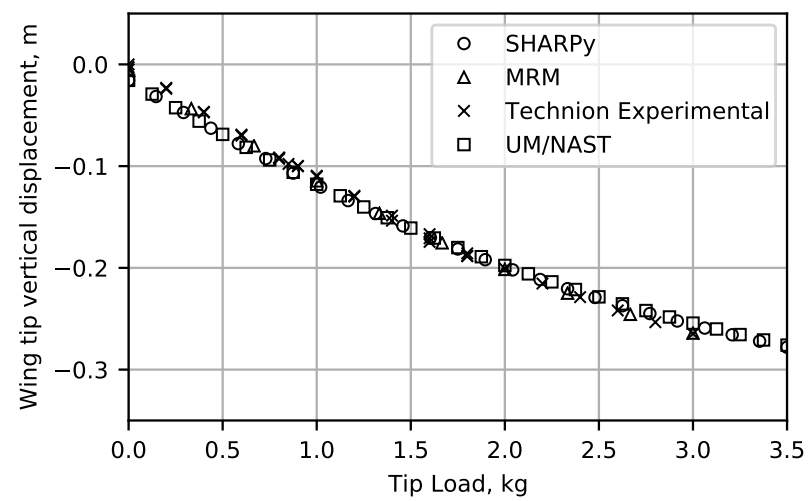

(a) Skin on

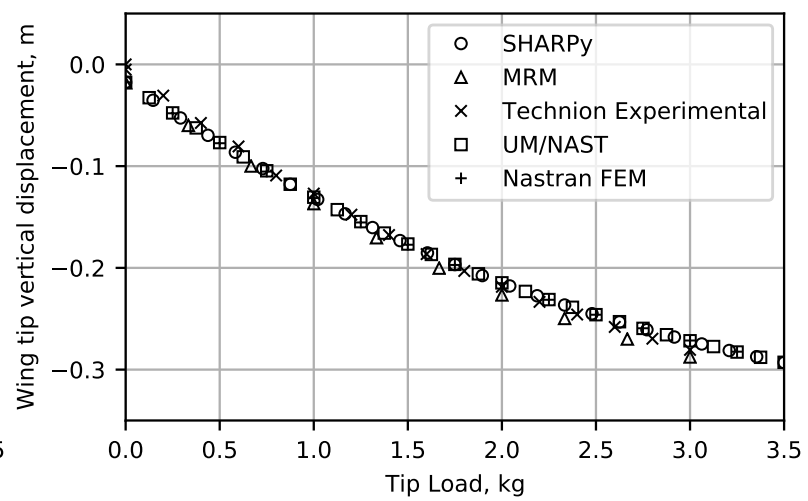

(b) Skin off

Fig. 6 Wing tip, mid-chord vertical displacement comparison under a twisting dead load between SHARPy, MRM, UM/NAST and Technion's experimental results.

\section{Steady aeroelastic simulations}

This simulation replicates a wind tunnel test in which the wing is clamped vertically at a given angle of attack and placed in a steady, uniform flow. The results obtained from SHARPy use the nonlinear structural solver and UVLM with an infinite horseshoe wake with symmetry boundary conditions at the wing root, such that there are no aerodynamic tip effects. Several angles of attack and free stream velocities are tested, with full simulation details in Table 4 . These are compared against the MRM model, UM/NAST results and wind tunnel tests. 


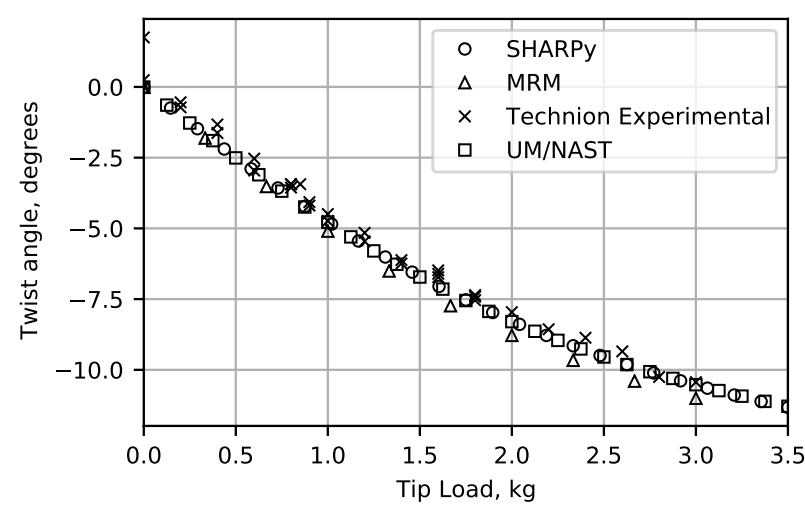

(a) Skin on

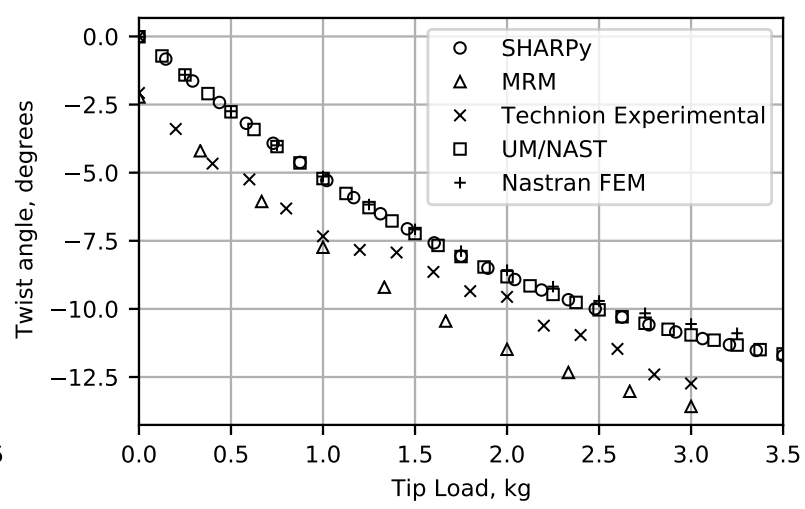

(b) Skin off

Fig. 7 Wing tip twist angle under a tip load (negative twist indicates leading edge down).

Table 4 SHARPy Steady aeroelastic simulation conditions.

\begin{tabular}{lc}
\hline Angle of attack & $5^{\circ}, 7^{\circ}$ \\
Free stream velocity & $1-60 \mathrm{~m} / \mathrm{s}$ \\
Density & $1.225 \mathrm{~kg} / \mathrm{m}^{3}$ \\
Chordwise panels & 16 \\
Spanwise panels & 32 \\
Wake panels & Infinite horseshoe \\
\hline
\end{tabular}

Figure 8 shows the wing tip out-of-plane displacements expressed in an inertial frame of reference with origin at the wing root and aligned with the flow (the structure rotates to simulate the angle of attack). The displacement shows a linear relationship with dynamic pressure below $1000 \mathrm{~Pa}(40.4 \mathrm{~m} / \mathrm{s})$ and in this region the results from all computational models and the experimental results are in good agreement. Once the geometrical nonlinearities become more apparent, the MRM displays a slightly more compliant response with the displacement approximately $5 \%$ above that of SHARPy at $60 \mathrm{~m} / \mathrm{s}$. The number of experimental data points gathered at high speeds is not sufficient to draw any conclusive comments on the fidelity of aeroelastic models, although the general trend is enough to have confidence in the results obtained at these speeds.

\section{Dynamic Results}

The previous results show good agreement between the SHARPy model, MRM, the FEM and the experimental results for static cases. To verify the inertia properties of the model, we will compare the vibration modes of the deformed wing under a follower force. Then, we proceed with a flutter prediction analysis for the wing in the wind tunnel fixed in a vertical position at various angles of attack to evaluate the variation in flutter speed. For these analysis, we additionally consider the case with a $10 \mathrm{~g}$ mass at the trailing edge of the wing tip section to evaluate its impact. This mass is modeled as a lumped, point mass with no inertia.

\section{A. Deformed wing modal analysis}

A modal analysis is performed on the deformed Pazy wing by placing a vertical follower force at the wing tip node. This illustrates the change in the natural modes of vibration of the structure as the wing deforms, which will have a significant impact on the flutter mechanics, as will be seen in the next subsection. The applied follower force at the wing tip node is aligned with the local vertical axis, therefore, in the linearisation of the structural system there are stiffening terms that arise due to the dependency of this force on perturbations to the structural degrees of freedom (by means of changes to the frame of reference on which it is defined) [23]. 


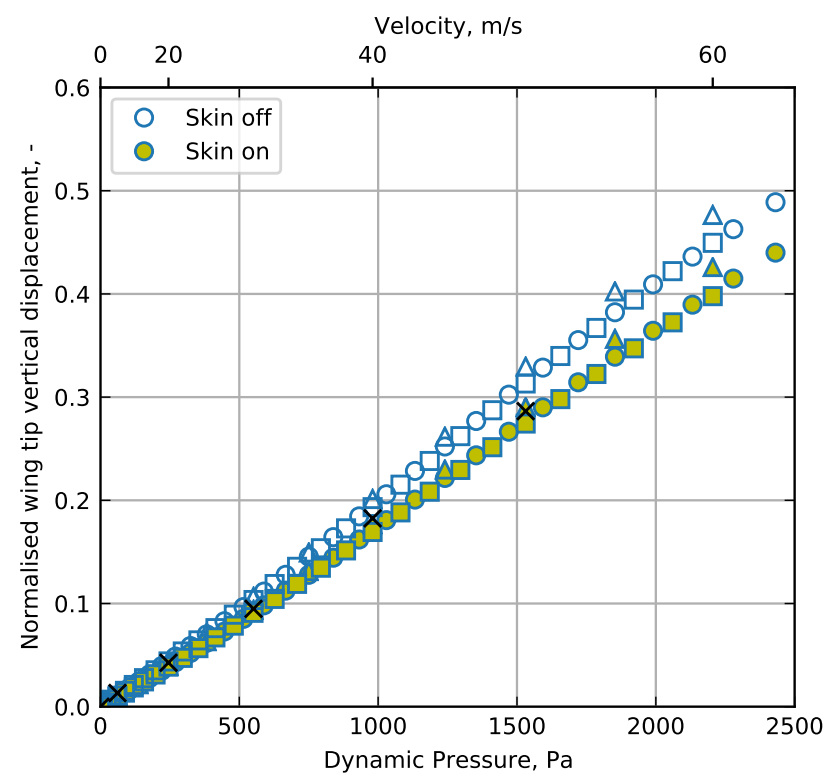

(a) 5 degrees

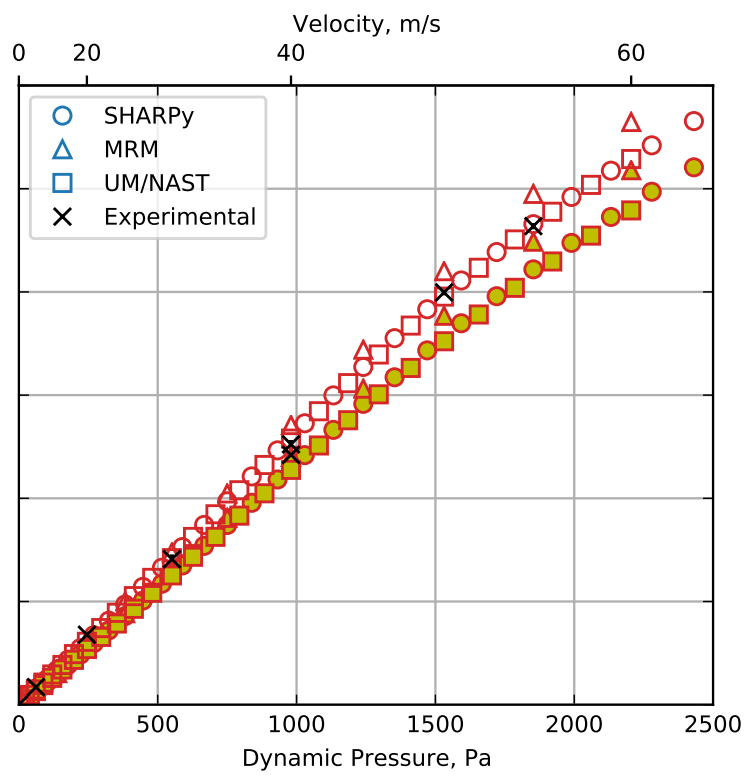

(b) 7 degrees

Fig. 8 Wing tip out-of-plane deflections at various angles of attack and free-stream velocities.

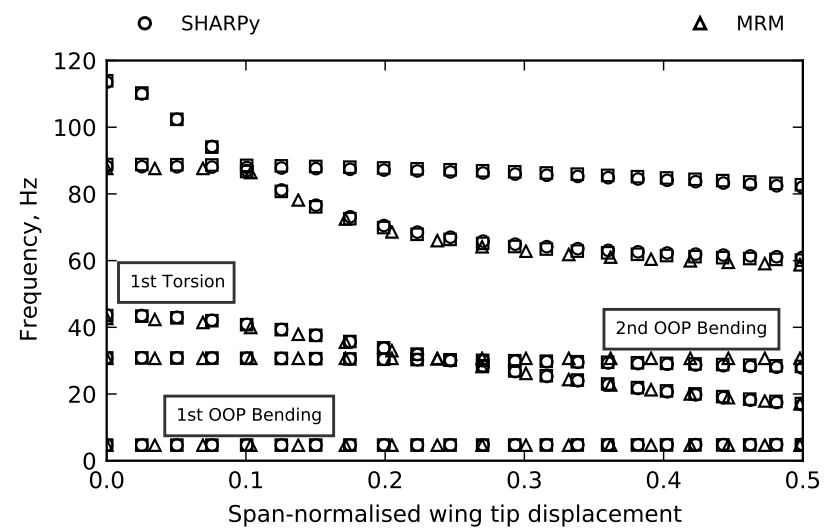

(a) Skin on
口 UM/NAST + NASTRAN

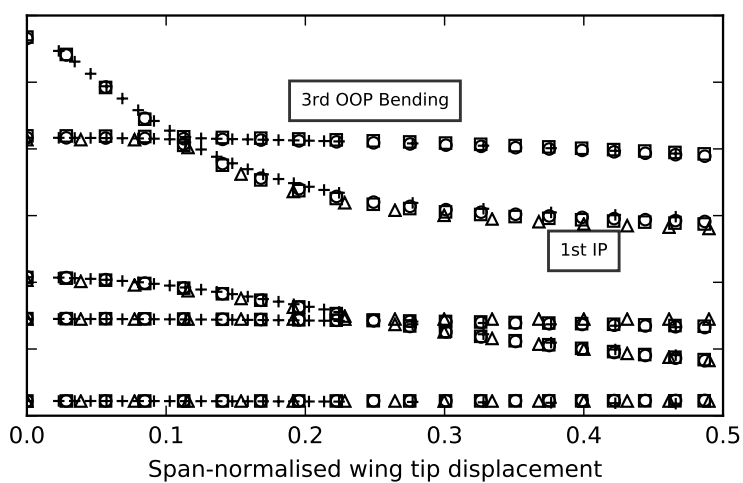

(b) Skin off

Fig. 9 Linearised beam's modes of vibration frequencies at a deformed condition when subject to a wing tip vertical follower force. 
Figure 9 compares the deformed structural frequencies between SHARPy, MRM, NASTRAN and UM/NAST [29] for both the skin on and off cases. The structural change of shape, in this case parametrised by the vertical wing tip deformation, causes two clear mode switches. Of particular importance is the drop in frequency of the 1st torsional mode, which eventually crosses the 2nd out-of-plane bending mode, and the drop in frequency of the 1st in-plane mode. The out-of-plane bending modes remain fairly constant in frequency, with the OOP1 seeing a slight increase in frequency caused by the stiffening terms arising from the linearisation of the wing tip follower force. We note that these results are only indicative as aerodynamic loading produces different equilibrium shapes to these and thus would skew the frequency against wing tip displacement relation.

\section{B. Zero angle of attack wing flutter prediction}

At zero degree angle of attack, there is no structural deformation with increasing wind speed. Therefore, the flutter boundary of the wing can be predicted using a conventional linear analysis as those traditionally employed by commercial software like NASTRAN. The aeroelastic system is linearised about the straight wing shape, as detailed in Sec. II.A. resulting in a linear UVLM and linear beam model. The UVLM is non-dimensionalised with respect to the free-stream flow, making it independent of velocity [19] while translating that dependency in velocity into a time scaling in the structural model. Consequently, a single aerodynamic model can be built which is valid across all incompressible flight velocities (as in the doublet-lattice method). This is then coupled to a structural model that needs updating at each wind speed condition through its mass and stiffness matrices, that had been scaled with the reference time (dependent on the free stream velocity). Note that updating the structural model at every wind speed is significantly more efficient than updating the linear UVLM. This is due to the required discretisation to achieve convergence which leads to UVLM systems of $10^{5}$ states, compared to the structural system's dimension, which is twice number of structural modes retained, in this case only six modes.

However, as explained in Sec. II the linear UVLM system is reduced using a Krylov sub-space method, which matches the steady state gain of the system's transfer functions. The resulting aeroelastic state-space model is small (42 states: 30 aerodynamic, 12 structural), and can be quickly evaluated for the desired wind speeds. The stability analysis is performed by computing the eigenvalues of the system, shown in Fig. 10 , between 10 and $120 \mathrm{~m} / \mathrm{s}$ at a density of $1.225 \mathrm{~kg} / \mathrm{m}^{3}$. In SHARPy the wing is discretised with 16 chordwise panels, 32 spanwise panels (16 3-noded finite element beams) and a 16-chord wake.

The MRM undeformed flutter prediction is based on a $k$ method analysis that uses the modal structural properties and the generalized aerodynamic matrices obtained from Zaero, It is validated with a $g$ method analysis conducted internally in Zaero.

It is useful to compare skin on against skin off flutter mechanics to evaluate the sensitivity to structural properties and, in particular, stiffness. This can highlight a suitable range of uncertainty of the flutter speed to inaccuracies in the beam models. The major contribution from the skin appears in the stiffness properties, in particular the out-of-plane bending stiffness which is, on average along the span, $11 \%$ larger than in the skin off model. Also, due to the linear nature of the constitutive relations of the beam models, the contribution to the stiffness will likely be overestimated, as these models are unable to capture the local buckling of the skin on the suction side as soon as the wing deforms. Thus, by comparing these two models we obtain a range of results where we expect to find the experimental ones.

The stability analysis for the wing at zero angle of attack in Fig. 10] shows that the increased airspeed causes a reduction in frequency of the torsional mode (T1), whose eigenvalue coincides with the second out-of-plane (OOP2) bending mode and results in the first flutter instability. This occurs both in the skin on and skin off models at very similar speeds, listed in Table 5, since the added stiffness of the skin-on case (predominantly in out-of-plane) increases the zero-velocity separation between OOP 2 and $\mathrm{T} 1$ by just $0.5 \mathrm{~Hz}(4 \%)$, which translates into a flutter speed increase of $3.1 \%$ between both cases.

Beyond this first instability, the torsional mode continues to drop in frequency, causing the flutter mode to cross back over to the left-hand plane (the "hump" in Fig. 10p. However, prior to the first flutter mode crossing back over the imaginary axes, a second flutter instability has occurred, this time a coupled torsion and first out-of-plane bending mode. Additionally, we explore the effect of including the trailing edge mass with the stability properties shown in Fig. 11. With the mass added, the torsional mode is damped to the point that it doesn't cross the imaginary axis, hence the first flutter mode is suppressed. However, after T1 switches with OOP2, it doesn't go as far into the left-hand-plane in Fig. 11p, thus as airspeed keeps increasing the T1-OOP1 onset of flutter speed is reduced from 93 and $98 \mathrm{~m} / \mathrm{s}$ for the skin off and skin on models respectively, to $84 \mathrm{~m} / \mathrm{s}$ for both cases, with the same result using the MRM.

To sum up, for the straight wing flutter in the nominal condition, Table 5 outlines the flutter speed at zero degrees 


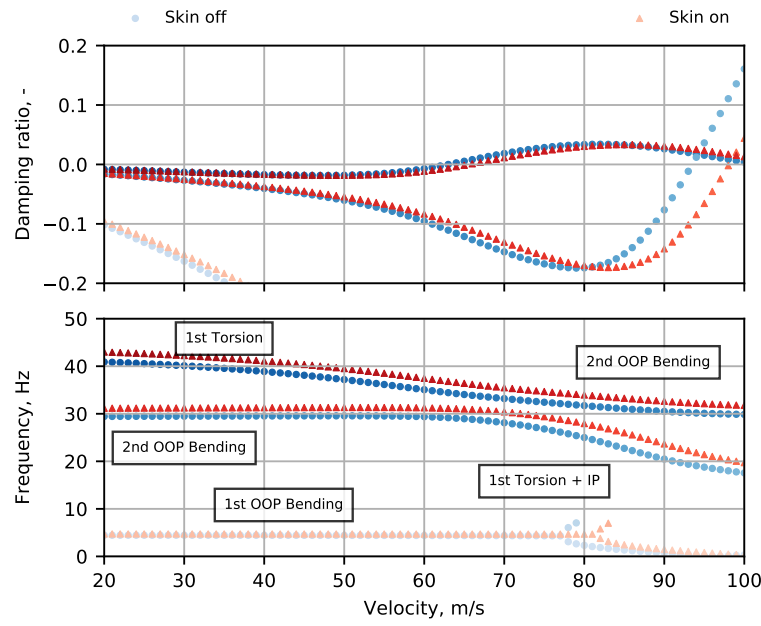

(a) Frequency-damping.

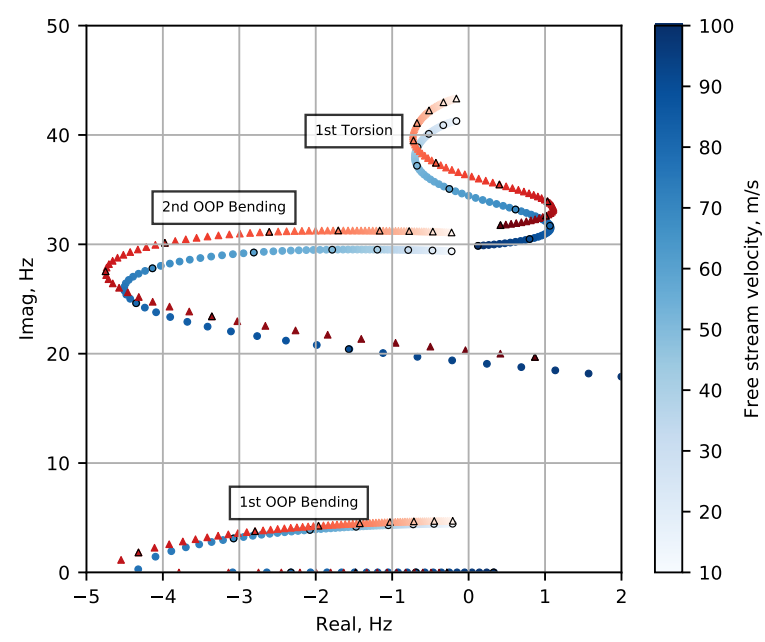

(b) Eigenvalues. Highlighted eigenvalues at $10 \mathrm{~m} / \mathrm{s}$ intervals.

Fig. 10 Stability of the Pazy wing at zero angle of attack. The frequency-damping-velocity (a) and eigenvalue plot (b) with eigenvalues highlighted in $10 \mathrm{~m} / \mathrm{s}$ intervals

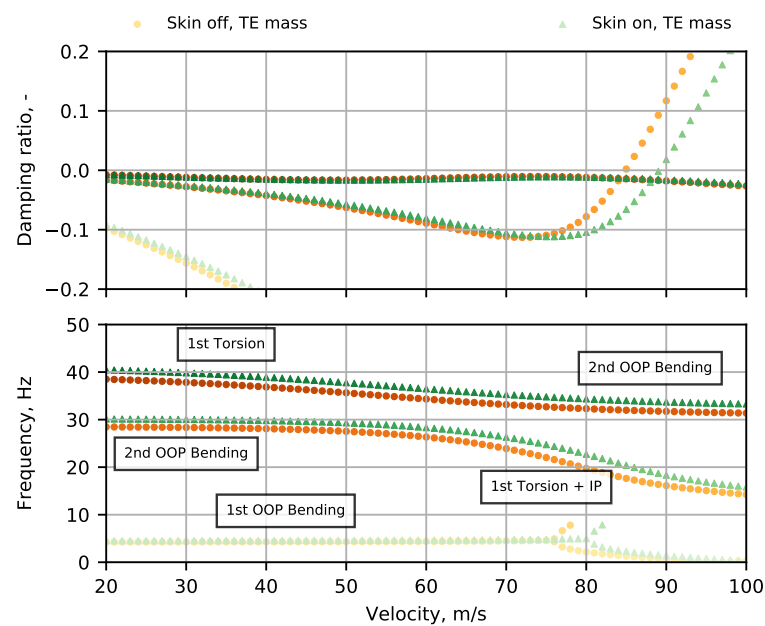

(a) Frequency-damping.

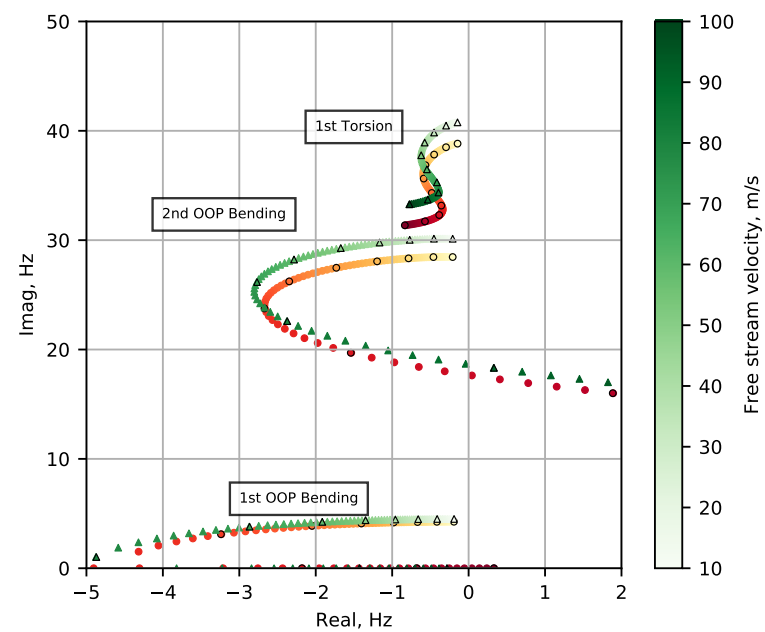

(b) Eigenvalues. Highlighted eigenvalues at $10 \mathrm{~m} / \mathrm{s}$ intervals.

Fig. 11 Stability of the Pazy wing at zero angle of attack. The frequency-damping-velocity (a) and eigenvalue plot (b) with eigenvalues highlighted in $10 \mathrm{~m} / \mathrm{s}$ intervals 
obtained using SHARPy and Zaero/MRM.

Table 5 Flutter speed of the wing at zero angle of attack.

\begin{tabular}{lcc}
\hline & Nominal & Trailing edge mas $\mathrm{S}^{\dagger}$ \\
SHARPy with skin & $65.0 \mathrm{~m} / \mathrm{s}$ & $84.0 \mathrm{~m} / \mathrm{s}$ \\
SHARPy without skin & $63.0 \mathrm{~m} / \mathrm{s}$ & $89.1 \mathrm{~m} / \mathrm{s}$ \\
Zaero / MRM UM beam with skin & $64.4 \mathrm{~m} / \mathrm{s}$ & \\
Zaero / MRM UM beam without skin & $61.8 \mathrm{~m} / \mathrm{s}$ & \\
Zaero / MRM NASTRAN model & $62.4 \mathrm{~m} / \mathrm{s}$ & $84.0 \mathrm{~m} / \mathrm{s}$ \\
\hline
\end{tabular}

\section{Flutter prediction for the deformed wing}

Predicting the flutter speed for the deformed wing configuration is a more complex problem, given that the assumption made during the linearization of the structural equations in particular (small deformations) no longer holds. This is clearly shown in Fig. 8 where, for example, the deflection of the wing set at 5 degrees angle of attack changes from $10 \%$ to $30 \%$ of span between 30 and $50 \mathrm{~m} / \mathrm{s}$. In addition, the dynamics of the structure change significantly with the deformed shape, as it was previously shown in Fig. 9 .

Therefore, computing the flutter speed of the deformed wing becomes an iterative procedure. First, the system is linearized about an initial guess value for the flutter speed and the flutter speed is estimated using the linear system as described in the previous section. Then, the nonlinear equilibrium condition at this computed flutter speed is found, the system linearized and the flutter speed estimated again. This iteration continues until the flutter speed estimation using linear methods converges to the speed at which that system was linearized and its nonlinear shape computed. We will refer to this point as the matched flutter point. The flutter prediction has been under the same conditions as those in the previous section.

In the case of the MRM, a linearized non-matched flutter solution was conducted at various velocities and angles of attack. The flutter solution is performed per angle of attack and velocity due to the effect on the geometry. The flutter margin is the margin between the flutter velocity and the velocity in the analysis. The matched point flutter velocity is found by finding the point at witch the calculation velocity and the flutter velocity are equal. These points coincide with the set-up in SHARPy. The analysis used the "fully modal" MRM approach, where the solution is based only on modal inertial and aerodynamic matrices, without an explicit representation of the two.

To illustrate the effect of deformation on flutter (by means of changing the root angle of attack), the stability plots for two angles of attack are shown in Fig. 12] and 13 for the skin off and skin on models, respectively. The intent is to use both models to have a plausible flutter boundary given the uncertainty introduced through the modelling of the Oralight skin.

The first effect angle of attack, and thus increased deformation, has on stability is to decrease the flutter speed. At higher angles of attack, the torsional mode observes a much steeper drop in natural frequency which advances the coalescence with the second out-of-plane bending mode. In addition, the cross-over of the two modes occurs at a lower speed and the first unstable flutter region is much shorter, as shown by the narrowing "hump" in the positive damping ratio region. Contrarily to the case at zero angle of attack, this shortening of the hump caused by the wing deformation results in a region of stability beyond the first flutter speed.

The second flutter mechanism follows a similar trend: the increased deformation due to a larger angle of attack brings forward the coalescence of the torsional and first out-of-plane bending modes, reducing the onset of instability speed.

Figure 14 summarises these two effects by showing the unstable flutter regions, one for each flutter mechanism, and the contours of root angle of attack show the wing deformation as the airspeed increases. It can be seen how increasing the root angle of attack brings forward the first flutter instability, albeit also making the region instability considerably smaller (i.e. narrowing the "hump"). This is because at higher angles of attack, a smaller increase in velocity is required to increase the deflection such that the aeroelastic torsional mode crosses towards the left-hand plane the imaginary axis.

\footnotetext{
$\dagger$ The flutter instability with the trailing edge mass attached corresponds to a first bending, first torsion flutter, as opposed to the nominal case in which flutter is a first torsion, second out-of-plane mode.
} 
- $\alpha=1.0^{\circ}$
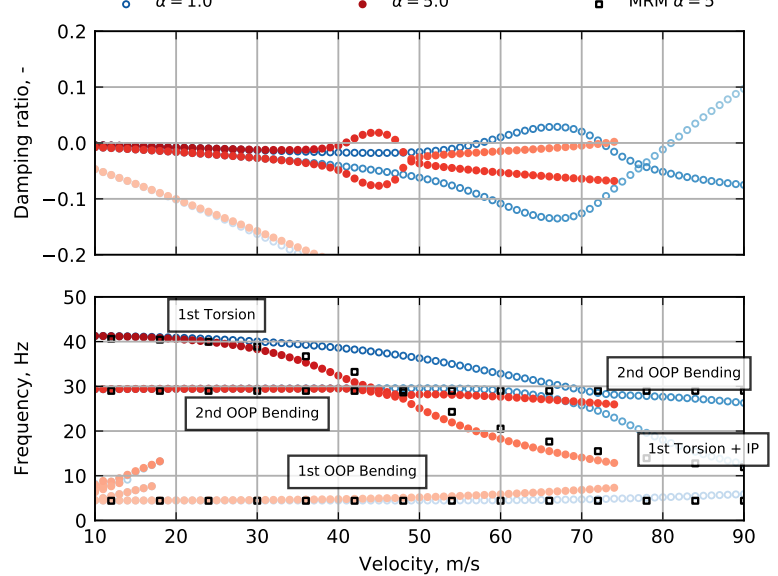

(a) V-g-omega plot, where colour scale relates to the natural frequency of the aeroelastic mode. MRM data shown for the 5 degree angle of attack case.

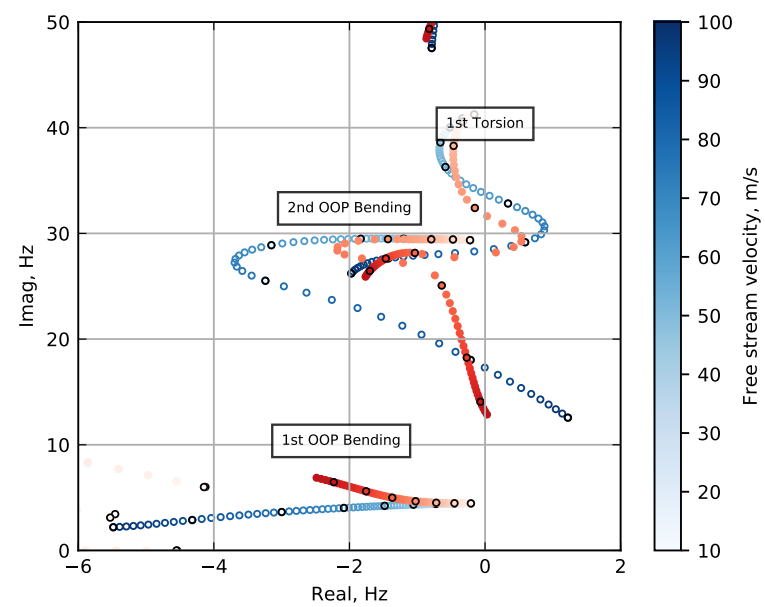

(b) Eigenvalues of the aeroelastic system. Eigenvalues are highlighted at $10 \mathrm{~m} / \mathrm{s}$ increments.

Fig. 12 SHARPy stability plot for the skin off wing fitted at 1 and 5 degrees angle of attack.

This is illustrated by the steep "stabilisation boundary" of the first flutter mode, which is fairly constant around $25 \%$ wing tip span wise deformation.

The second flutter speed is also shown to decrease with increased deflection and angle of attack, although this decrease is not monotonic and appears to even increase beyond 5 degrees angle of attack. These regions for the skin off model provide the most conservative flutter scenario for a wind tunnel test, and could offer insight into an experiment to reach the second flutter instability region without excessive deflections and spending minimal time in the first flutter region.

Turning to the comparison between codes, it is in these deformed, dynamic cases where the difference in aerodynamic models becomes most obvious. The MRM frequency predictions in Fig. $12 \mathrm{a}$ and $13 \mathrm{~h}$ for the 5 degree angle of attack show an excellent agreement at the lower velocities, where the deflection is smallest. Beyond $50 \mathrm{~m} / \mathrm{s}$, point at which the deflection reaches $30 \%$ of the span, the differences in the T1 and OOP1 start becoming noticeable. This can be attributed to the change of dihedral effect caused by the deformation, which is captured by the linearised SHARPy UVLM but not the MRM implementation of the linearised aerodynamic system. This effect has also been previously observed by the authors on the setting of a different framework [21] that used the UVLM linearised at the straight wing condition as aerodynamic model. In such framework, it was observed that the OOP1 reduced its frequency to become a purely real mode as deformation increased, as opposed to using the deformed condition as reference for the linearised UVLM, case in which the OOP1 frequency increases with deformation as seen here.

The difference is further observed in Fig. 14, where the agreement in the flutter boundary is excellent for the small deformation conditions (the first flutter instability in Fig. 14a) as opposed to the second flutter mode shown which differs substantially at the larger wing tip deflections.

\section{Conclusions}

We have evaluated and compared between two different aeroelastic toolboxes, SHARPy and Technion's MRM, the structural static and dynamic response of the Pre-Pazy wing. The agreement between tools for the different models and correlation with experimental results give confidence in using these models to support the design of non-destructive wind tunnel experiments to explore the wing's flutter mechanisms. The two identified flutter modes' dependency on the wing deformation provide an excellent example to showcase the need for nonlinear aeroelastic analysis tools. Both the MRM and SHARPy capture well these effects, although at high deformations the need for aerodynamic models linearised at the deformed condition become of paramount importance to predict flutter.

On the other hand, the complex modelling of the Oralight skin introduces some uncertainties into these analyses which, thus far, have been tackled by using two extreme models. By using skin on and skin off models it is expected that 

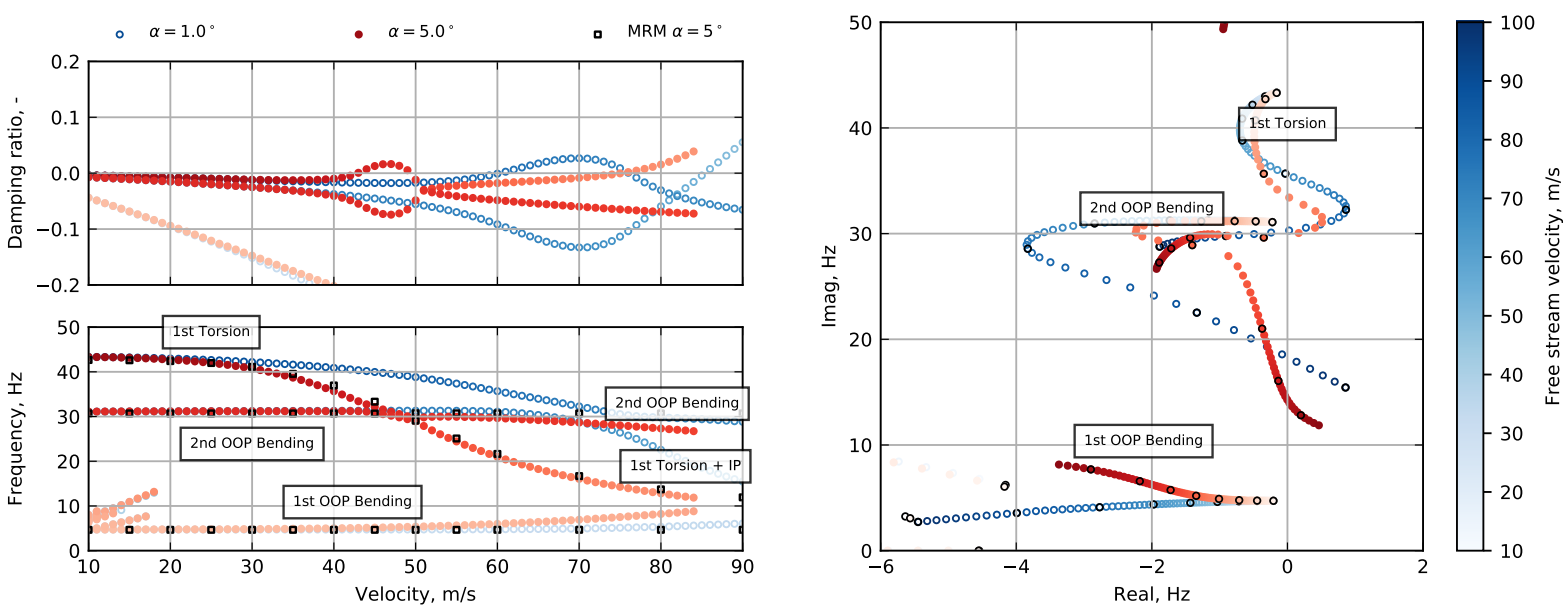

(a) V-g-omega plot, where colour scale relates to the natural (b) Eigenvalues of the aeroelastic system with eigenvalues frequency of the aeroelastic mode. MRM data shown for the highlighted at $10 \mathrm{~m} / \mathrm{s}$ increments. 5 degree angle of attack case.

Fig. 13 SHARPy stability plot for the wing fitted at 1 and 5 degrees angle of attack.

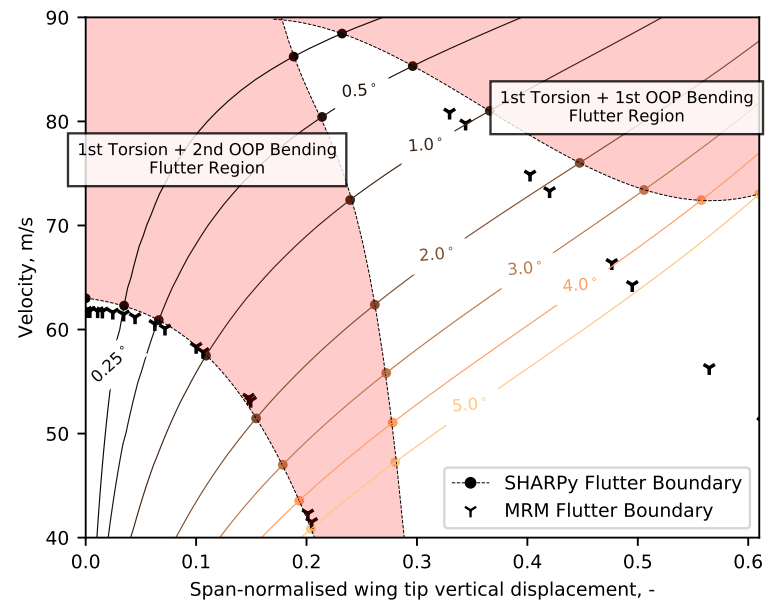

(a) Skin off

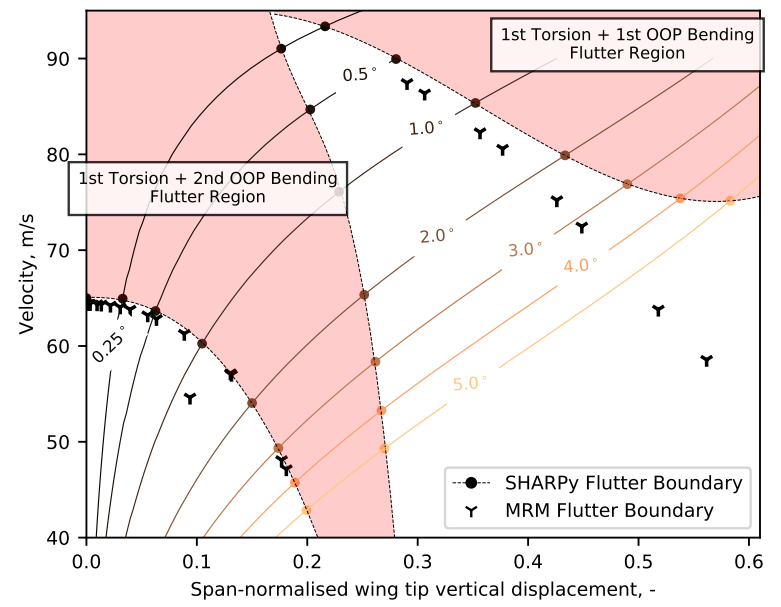

(b) Skin on

Fig. 14 Flutter boundaries and regions, with contours showing wing tip deflection at a given root angle of attack. 
the results show the two limits between which the flutter boundary is expected in the wind tunnel experiments.

Further work will focus on the analysis of an updated computational model of the Pazy wing, the comparison against dynamic wind tunnel tests and other numerical tools that also consider the Pazy example [29, 30].

\section{Research Data}

With the objective of making this a benchmark case that can be used to validate and test future aeroelastic simulation tools, the SHARPy model, scripts and results are all made available online. The results presented herein can be downloaded from the Zenodo archive.

Table 6 Online repositories for models, scripts and results.

\begin{tabular}{l|l}
\hline & URL \\
\hline AePW3 Results & https://doi.org/10.5281/zenodo.4311606 \\
\cline { 2 - 2 } SHARPy simulation scripts & https://doi.org/10.5281/zenodo.4299819 \\
\hline
\end{tabular}

\section{Acknowledgements}

The authors would like to gratefully acknowledge the Pazy Foundation for their support of the Pazy-Wing experimental campaign.

The authors would also like to thank Markus Ritter from the DLR for leading and organising the Large Deflection working group within the 3rd Aeroelastic Prediction Workshop.

Norberto Goizueta would like to thank Mark Korchma from Imperial College for deriving the original equivalent beam model for SHARPy that started this work and Cristina Riso for her help providing the UM/NAST model.

The Department of Aeronautics at Imperial College has sponsored Norberto Goizueta's research under EPSRC grant EP/R513052/1.

\section{References}

[1] "Airbus Zephyr Solar High Altitude Pseudo-Satellite flies for longer than any other aircraft during its successful maiden flight," , 2018. URL https://www.airbus.com/newsroom/press-releases/en/2018/08/Airbus-Zephyr-SolarHigh-Altitude-Pseudo-Satellite-flies-for-longer-than-any-other-aircraft.html. Accessed 05/06/2020.

[2] "Odysseus - Global Reach, Airborne for Months, Powered by the Sun,", 2020. URL https://www . aurora . aero/odysseushigh-altitude-pseudo-satellite-haps/. Accessed 05/06/2020.

[3] "Persistent High Altitude Solar Aircraft (PHASA-35®) has the potential to stay airborne for a year," , 2020. URL https://www. baesystems . com/en/article/ground-breaking-solar-powered-unmanned-aircraftmakes-first-flight. Accessed 05/06/2020.

[4] “Skydweller,”, 2020. URL https://skydweller.aero Accessed 29/05/2020.

[5] Noll, T., Brown, J., Perez-Davis, M., Ishmael, S., Tiffany, G., and Gaier, M., "Investigation of the Helios Prototype Aircraft Mishap,” Tech. Rep. January, NASA, 2004. https://doi.org/10.1109/MWSYM.2002.1012130, URL https://www.nasa.gov/pdf/ 64317main_helios.pdf

[6] d'Oliveira, F. A., de Melo, F. C. L., and Devezas, T. C., "High-Altitude Platforms - Present Situation and Technology Trends," Journal of Aerospace Technology and Management, Vol. 8, No. 3, 2016, pp. 249-262.

[7] Murua, J., Palacios, R., and Graham, J. M. R., "Applications of the unsteady vortex-lattice method in aircraft aeroelasticity and flight dynamics,” Progress in Aerospace Sciences, Vol. 55, 2012, pp. 46-72. https://doi.org/10.1016/j.paerosci.2012.06.001

[8] del Carre, A., Teixeira, P., Palacios, R., and Cesnik, C. E., "Nonlinear response of a very flexible aircraft under lateral gust," International Forum on Aeroelasticity and Structural Dynamics 2019, IFASD 2019, 2019, pp. 1-27.

[9] Tang, D., and Dowell, E. H., "Experimental and Theoretical Study on Aeroelastic Response of High-Aspect-Ratio Wings," AIAA Journal, Vol. 39, No. 8, 2001, pp. 1430-1441. https://doi.org/10.2514/2.1484 URL https://doi.org/10.2514/2.1484. 
[10] Avin, O., Drachinsky, A., Ben-Shmuel, Y., and Raveh, D. E., "Design of an Experimental Benchmark of a Highly Flexible Wing," 60th Israel Annual Conference on Aerospace Sciences, 2020, pp. 1-18.

[11] Carre, A., Muñoz-Simón, A., Goizueta, N., and Palacios, R., "SHARPy : A dynamic aeroelastic simulation toolbox for very flexible aircraft and wind turbines," Journal of Open Source Software, Vol. 4, No. 44, 2019, p. 1885. //doi.org/10.21105/joss.01885

[12] Drachinsky, A., and Raveh, D. E., "Modal Rotations: A Modal-based Method for Large Structural Deformations," AIAA Journal, 2020. https://doi.org/10.2514/1.J058899.

[13] Geradin, M., and Cardona, A., Flexible multibody dynamics: a finite element approach, John Wyley and Sons, Chichester, 2001.

[14] Simpson, R. J. S., and Palacios, R., "Numerical aspects of nonlinear flexible aircraft flight dynamics modeling," 54th AIAA/ASME/ASCE/AHS/ASC Structures, Structural Dynamics, and Materials Conference, 2013, pp. 1-25. https://doi.org/10. 2514/6.2013-1634 URL http://arc.aiaa.org/doi/10.2514/6.2013-1634.

[15] Hesse, H., Palacios, R., and Murua, J., "Consistent structural linearisation in flexible aircraft dynamics with large rigid-body motion,” AIAA Journal, Vol. 52, No. 3, 2014, p. 528. https://doi.org/10.1016/j.compstruc.2012.05.011

[16] Katz, J., and Plotkin, A., "Unsteady Incompressible Potential Flow," Low Speed Aerodynamics, Cambridge University Press, 2001, Chap. 13, $2^{\text {nd }}$ ed., pp. 369-433.

[17] Del Carre, A., and Palacios, R., "Efficient Time-Domain Simulations in Nonlinear Aeroelasticity," AIAA Scitech Forum, 2019, pp. 1-20. https://doi.org/10.2514/6.2019-2038

[18] Simpson, R. J. S., Palacios, R., and Murua, J., "Induced-Drag Calculations in the Unsteady Vortex Lattice Method," AIAA Journal, Vol. 51, No. 7, 2013, pp. 1775-1779. https://doi.org/10.2514/1.j052136

[19] Maraniello, S., and Palacios, R., "State-Space Realizations and Internal Balancing in Potential-Flow Aerodynamics with Arbitrary Kinematics," AIAA Journal, Vol. 57, No. 6, 2019, pp. 1-14. https://doi.org/10.2514/1.J058153. URL https: //arc.aiaa.org/doi/10.2514/1.J058153

[20] Murua, J., Martínez, P., Climent, H., van Zyl, L., and Palacios, R., “T-tail flutter: Potential-flow modelling, experimental validation and flight tests," Progress in Aerospace Sciences, Vol. 71, 2014, pp. 54-84. https://doi.org/https://doi.org/10.1016/j. paerosci.2014.07.002 URL http://www.sciencedirect.com/science/article/pii/S0376042114000669.

[21] Artola, M., Goizueta, N., Wynn, A., and Palacios, R., "Modal-Based Nonlinear Estimation and Control for Highly Flexible Aeroelastic Systems," AIAA Scitech Forum, 2020, pp. 1-23. https://doi.org/10.2514/6.2020-1192

[22] Maraniello, S., and Palacios, R., "Parametric Reduced-Order Modeling of the Unsteady Vortex-Lattice Method," AIAA Journal, Vol. 58, No. 5, 2020, pp. 2206-2220. https://doi.org/10.2514/1.j058894

[23] Goizueta, N., Wynn, A., and Palacios, R., "Parametric Krylov-based order reduction of aircraft aeroelastic models," AIAA Scitech Forum, 2021.

[24] Drachinsky, A., and Raveh, D. E., "Nonlinear Aeroelastic Analysis of Highly Flexible Wings Using the Modal Rotation Method," AIAA Scitech Forum, 2021.

[25] Avin, O., Drachinsky, A., Ben Shmuel, Y., and Raveh, D. E., "An Experimental Benchmark of a Very Flexible Wing," AIAA Scitech Forum, 2021.

[26] Riso, C., and Cesnik, C. E. S., "Equivalent Beam Distributions of the Pazy Wing,” Tech. rep., University of Michigan, 2020.

[27] Zaero Theoretical Manual V. 9.2, Scottsdale, AZ, $3^{\text {rd }}$ ed., 2017.

[28] Avin, O., Drachinsky, A., Ben-Shmuel, Y., and Raveh, D. E., “An Experimental Benchmark of a Highly Flexible Wing,” AIAA Scitech Forum, 2021.

[29] Riso, C., and Cesnik, C. E. S., "UM/NAST Experimental Validation Using the Pazy Wing Aeroelastic Benchmark," AIAA Scitech Forum, 2021.

[30] Ritter, M., Hilger, J., and Zimmer, M., "Static and Dynamic Simulations of the Pazy Wing Aeroelastic Benchmark by Nonlinear Potential Aerodynamics and detailed FE Model,” AIAA Scitech Forum, 2021. 